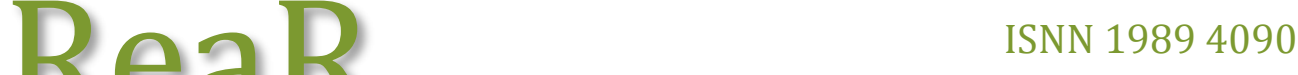 \\ Revista electrónica de AnestesiaR
}

\section{La ecografía como método simple y rápido para evaluar la correcta colocación de sondas de alimentación enteral en pacientes críticos con ventilación mecánica}

Artículo original: Nedel WL, Jost MNF, Filho JWF. A simple and fast ultrasonographic method of detecting enteral feeding tube placement in mechanically ventilated, critically ill patients. J Intensive

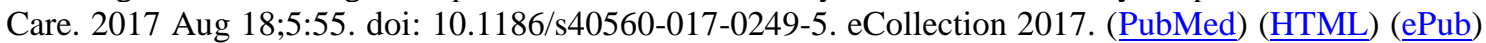
(PDF)

León Guisado A (1), Serna Gandía MB (1), Reguera Rosal J (2).

(1) Hospital Marina Salud Denia, Alicante.

(2) Hospital IMED-Levante. Benidorm, Alicante.

\section{Resumen}

La radiografía $(\mathrm{Rx})$ abdominal, el método diagnóstico utilizado para verificar la posición de la sonda de alimentación enteral (SAE), es una fuente de irradiación y de potenciales efectos adversos para los pacientes. Hay pocos estudios sobre colocación de SAE y ecografía. Los autores estudiaron 41 pacientes con inserción de SAE y mantenimiento de la guía hasta la comprobación de su correcta colocación con ecografía, detectando una correcta colocación en 38 pacientes frente a 3 pacientes con una inadecuada posición de la SAE, con una sensibilidad del 97\% (IC 95\%: 84.9-99.8\%) y una especificidad del 100\% (IC 95\%: 19.7-100\%). La evaluación de la posición de la SAE a través del abdomen mediante ecografía es práctica y segura, asociándose con resultados diagnósticos satisfactorios.

\section{Introducción}

La radiografía (Rx) abdominal, el método diagnóstico utilizado para verificar la posición de la sonda de alimentación enteral (SAE), es una fuente de irradiación y de potenciales efectos adversos para los pacientes. Hay pocos estudios sobre colocación de SAE y ecografía. Los autores estudiaron 41 pacientes con inserción de SAE y mantenimiento de la guía hasta la comprobación de su correcta colocación con ecografía, detectando una correcta colocación en 38 pacientes frente a 3 pacientes con una inadecuada posición de la SAE, con una sensibilidad del 97\% (IC 95\%: 84.9-99.8\%) y una especificidad del 100\% (IC 95\%: 19.7$100 \%$ ). La evaluación de la posición de la SAE a través del abdomen mediante ecografía es práctica y segura, asociándose con resultados diagnósticos satisfactorios.

La nutrición enteral (NE) es la alimentación de elección en los pacientes críticos con función gastrointestinal preservada (1). Para la adecuada administración de la NE se debe colocar una sonda de alimentación enteral (SAE), requiriéndose frecuentemente una prueba de imagen para confirmar su correcto posicionamiento. La radiografía $(\mathrm{Rx})$ abdominal, el método diagnóstico gold estándar para comprobar la SAE, es una fuente de radiación para los pacientes y conlleva un potencial riesgo de retirada accidental de dispositivos, diseminación microbiana, costes adicionales y de un retraso en la obtención de la imagen $(2$, $3)$. Muchos procedimientos en las 
unidades de cuidados intensivos (UCIs) se llevan a cabo bajo control ecográfico (4), no obstante hay pocos estudios sobre colocación de SAE guiada por ecografía (2, 3). El objetivo de los autores es evaluar mediante un estudio prospectivo la efectividad de la ecografía como prueba de imagen para la correcta colocación de SAE en pacientes con VM, ya que es una técnica rápida y simple basada en la visualización de la guía en el interior de la SAE. El objetivo secundario es comparar el tiempo que se requiere para confirmar la correcta colocación con la $\mathrm{Rx}$ de abdomen con respecto a la ecografía.

\section{Método}

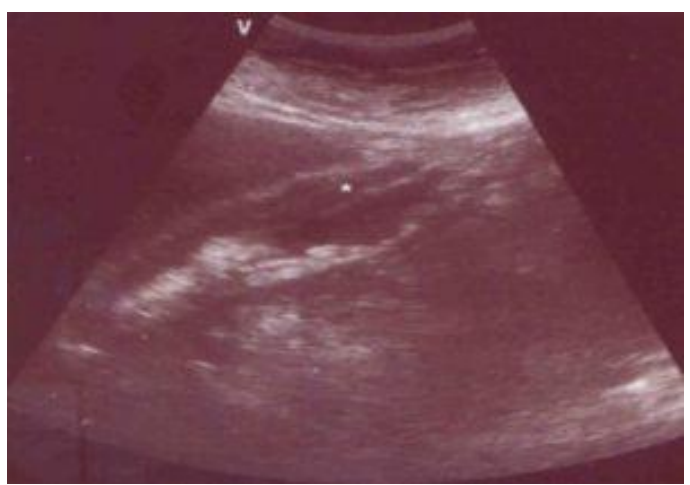

Figura 1: Área Gástrica y posición de la guía

Los autores evalúan de forma prospectiva pacientes en UCI con VM invasiva y colocación de SAE. En todos los casos, la longitud de la SAE se calculó en base a la distancia desde el apófisis xifoides hasta el lóbulo de la oreja y posteriormente hasta la nariz; la colocación se realizó mediante el uso de guía, que se mantuvo hasta el examen ecográfico, visualizándose como una imagen hiperecogénica.

La ecografía fue realizada por un facultativo no asistencial (M Turbo TM By Sonosite Fuji Film, Bothell, USA), y todas las $\mathrm{Rx}$ abdominales fueron analizadas prospectivamente por el equipo de UCI y de forma ciega al final del estudio de manera retrospectiva. El investigador principal, era un médico de
UCI con una experiencia previa mínima de 20 exámenes ecográficos para esta técnica. El paciente es estudiado con una sonda convexa de $3 \mathrm{MHz}$, realizándose un examen abdominal con cortes sagitales y transversos a nivel de la zona epigástrica. Si la SAE no era visualizada la sonda se orientaba hacia el hipocondrio izquierdo para visualizar la sonda gástrica, identificándose tanto el tracto digestivo como la guía, dentro del mismo (Fig. 1). No se registró en este estudio si la localización de la sonda era gástrica $o$ duodenal, solamente se registraba si estaba adecuadamente posicionada en el tracto digestivo, o fuera de este (colocación esofágica, nasofaríngea o en el árbol traqueobronquial). El tiempo entre la colocación de la SAE y el estudio ecográfico fue comparado con el tiempo transcurrido entre el procedimiento y el diagnóstico radiográfico, definido como el tiempo transcurrido entre la implantación de la SAE y la publicación de la imagen para evaluar su posicionamiento. Las variables continuas fueron expresadas como media y rango intercuartílico. El tiempo transcurrido por cada método fue comparado por el test de Wilcoxon. Sensibilidad, especificidad, valor predictivo positivo $\mathrm{y}$ negativo fueron calculados con un coeficiente de confianza del 95\%. El análisis estadístico fue realizado utilizando SPSS versión 20.0 (SPSS Inc., Chicago, USA). Un valor de $p<0,05$ fue considerado como estadísticamente significativo.

\section{Resultados}

Se analizaron 41 pacientes $(53 \%$ varones, $14 \%$ quirúrgicos). La duración del procedimiento ecográfico hasta la adecuada visualización de la guía fue de 90 segundos. Los principales datos clínicos están expresados en la Tabla 1. 


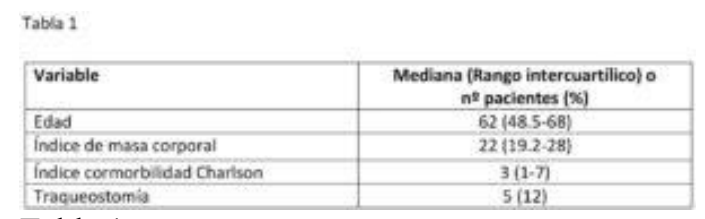

Tabla 1.

Con la Rx abdominal 39 pacientes presentaron una correcta colocación de la SAE (95\%), frente a 2 pacientes en los que se objetivó una colocación inadecuada. Con la ecografía, 38 pacientes presentaron una correcta colocación de la SAE frente a 3 pacientes en los que se objetivó una colocación inadecuada (Tabla 2), con una sensibilidad del 97\% (IC 95\%: 84,9-99,8\%), especificidad 100\% (IC 95\%: $19,7-100 \%$ ), valor predictivo positivo 100\% (IC 95\%: 88,5-100\%), valor predictivo negativo 66\% (IC 95\%: 12,5-98\%). El tiempo medio de la inserción de la SAE hasta el diagnóstica de la colocación fue de 46 minutos (20 a 163 minutos), mientras que el tiempo medio de la Rx abdominal fue de 162 minutos (98-247 minutos), $\mathrm{p}<0,0001$.

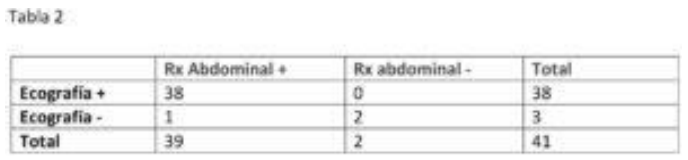

Tabla 2

\section{Discusión}

Los autores mediante la ecografía fueron capaces de valorar de forma rápida y sencilla la adecuada colocación de la SAE en la mayoría de los pacientes críticos con VM, confirmando la alta sensibilidad de este método en pacientes de UCI, incluso llevada a cabo por médicos sin una formación específica en ecografía. Este estudio añade nuevos datos a los previamente descritos en población pediátrica (5) con posterior extensión a los adultos y con resultados similares a los escasos estudios previos $(2,3)$. Habitualmente la SAE es detectada por su sombra acústica más que por la propia sonda, por lo que la técnica descrita, usando una guía, es más sencilla de reproducir. El principal objetivo era determinar la correcta colocación de la SAE en el tracto digestivo, no analizar su posicionamiento pre o post pilórico, que en la gran mayoría de los pacientes carece de importancia ya que no se observaran cambios en cuanto a la tolerancia gastrointestinal (6).

Este estudio tiene algunas limitaciones. A pesar de los buenos resultados con la ecografía y su menor tiempo para detectar el correcto posicionamiento de la sonda con respecto a la $\mathrm{Rx}$, hay que tomar estos datos con precaución. Cada UCI tiene distintas capacidades logísticas, tanto para el diagnóstico como para el inicio de la alimentación, y por tanto, el tiempo para realizar ambos exámenes pueden ser distintos al encontrado en este trabajo. Por ello, estos datos, no tienen validez externa. Posiblemente en otros escenarios el tiempo para el diagnóstico por ecografía también sea inferior al de $\mathrm{Rx}$ abdominal, aunque distintos a los encontrados en este estudio. La Rx sigue siendo el método estándar para la confirmación del posicionamiento de la SAE, y la ecografía se posiciona como una alternativa con la necesidad de realizar estudios aleatorizados prospectivos. Bajo el punto de vista de los autores la utilización de la ecografía disminuye la necesidad de $\mathrm{Rx}$ y las complicaciones derivadas de la misma.

\section{Conclusiones}

La evaluación de la correcta colocación de la SAE mediante ecografía abdominal es práctica y segura, siendo una técnica rápida que se asocia a una buena precisión diagnóstica.

\section{Bibliografía}

1. McClave SA, Taylor BE, Martindale RG, Warren MM, Johnson DR, Braunschweig C, et al. Guidelines for the provision and assessment 
of nutrition support therapy in the adult critically ill patient: Society of Critical Care Medicine (SCCM) and American Society for Parenteral and Enteral Nutrition (ASPEN). J Parenter Enter Nutr. 2016;40:159-211. (PubMed)

(HTML)

2. Gok F, Kilicaslan A, Yosunkaya A. Ultrasound-guided nasogastric feeding tube placement in critical care patients. Nutr Clin Pract. 2015;30:257-60. (ubMed) ( $\underline{\text { HTML) }}$

3. Vigneau C, Baudel JL, Guidet B, Offenstadt G, Maury E. Sonography as an alternative to radiography for nasogastric feeding tube location. Intensive Care Med. 2005;31:1570-2. ( $\underline{\text { PubMed) }}$ ( $\underline{\text { HTML}})$

4. Frankel HL, Kirkpatrick AW, Elbarbary M, Blaivas M, Desai H, Evans D, et al. Guidelines for the appropriate use of bedside general and cardiac ultrasonography in the evaluation of critically ill patients-part I: general ultrasonography. Crit Care Med. 2015;43:2479_ 502. (PubMed)
5. Greenberg M, Bejar R, Asser S. Confirmation of transpyloric feeding tube placement by ultrasonography. J Pediatr. 1993;122:413-5. (PubMed)

6. Jiyong $\mathrm{J}$, Tiancha $\mathrm{H}$, Huiquin $\mathrm{W}$, Jingfen $\mathrm{J}$. Effect of gastric versus post-pyloric feeding on the incidence of pneumonia in critically-ill patients: observations from traditional and Bayesian random-effects meta-analysis. Clin Nutr. 2013;32:8-15. (PubMed)

Correspondencia al autor

Antonia Leon Guisado tonlegui@hotmail.com

FEA Servicio de Anestesiología y Cuidados Intensivos.

Hospital Marina Salud Denia, Alicante.

Aceptado para blog en febrero de 2018. 